PROCEEDINGS OF THE

AMERICAN MATHEMATICAL SOCIETY

Volume 124, Number 6, June 1996

\title{
STABLE SPLITTINGS OF $\mathrm{BO}(2 n)$ AND BU $(2 n)$
}

\author{
DUNG YUNG YAN \\ (Communicated by Thomas Goodwillie)
}

\begin{abstract}
Using the Snaith-Mitchell-Priddy splittings of $\mathrm{BO}(2 n)$ and $\mathrm{BU}(2 n)$, we can give new stable splittings of $\mathrm{BO}(2 n)$ and $\mathrm{BU}(2 n)$ respectively.
\end{abstract}

In [5] and [6] Snaith, Mitchell, and Priddy showed that the natural filtrations on $\mathrm{BO}(n), \mathrm{BU}(n)$, and $\operatorname{BSP}(n)$ stably split, respectively, and in [2] Henn and Mui showed the corresponding splitting for $\mathrm{BSO}(2 n+1)$ and $\mathrm{BSU}(2 n+1)$. The purpose of this note is to give new stable splittings of $\mathrm{BO}(2 n)$ and $\mathrm{BU}(2 n)$, respectively.

Let $g_{2 n}: O(2 n) \rightarrow \mathrm{SO}(2 n+1)$ be defined by $g_{2 n}(\alpha)=\operatorname{det}(\alpha) \oplus \alpha$. Then we have

$$
B g_{2 n}: \quad \mathrm{BO}(2 n) \rightarrow \mathrm{BSO}(2 n+1) .
$$

Let $Y_{2 n}$ be the stable fibre of $B g_{2 n}$, that is,

$$
Y_{2 n} \stackrel{\delta_{2 n}}{\longrightarrow} \mathrm{BO}(2 n) \stackrel{B g_{2 n}}{\longrightarrow} \mathrm{BSO}(2 n+1)
$$

is a stable fibration.

Theorem A. Localized at the prime 2, the stable fibre $Y_{2 n}$ of $B g_{2 n}$ is a stable summand of the stable fibre $Y_{2 n+2}$ of $B g_{2 n+2}$.

Corollary B. Localized at the prime 2 , for each $n$ there are 2-local spectra $F_{2 i}$, $1 \leq i \leq n$, such that

$$
F_{2} \vee F_{4} \vee F_{6} \vee \cdots \vee F_{2 n} \rightarrow \mathrm{BO}(2 n) \stackrel{B g_{2 n}}{\rightarrow} \mathrm{BSO}(2 n+1)
$$

is a stable filtration and

$$
\mathrm{BO}(2 n) \cong \mathrm{BSO}(2 n+1) \vee F_{2} \vee F_{4} \vee F_{6} \vee \cdots \vee F_{2 n}
$$

Remark 1. In [3], Mitchell and Priddy proved that the homotopy type of $F_{2}$ is $\sum^{-2} \overline{S P^{4}} S^{0} \vee P_{1}^{\infty}$ (see [3]).

Remark 2. We have analogous theorems for $\mathrm{BU}(2 n)$ and $\mathrm{BSU}(2 n+1)$.

From now on we will suppress details for $\mathrm{BU}(2 n)$, which can be obtained easily from those for $\mathrm{BO}(2 n)$, and all spaces or spectra are implicitly localized at prime 2 .

Lemma 1. Let $Y_{2 n} \stackrel{\delta_{2 n}}{\longrightarrow} \mathrm{BO}(2 n) \stackrel{B g_{2 n}}{\rightarrow} \mathrm{BSO}(2 n+1)$ be a stable fibration. Then $\mathrm{BO}(2 n) \cong \mathrm{BSO}(2 n+1) \vee Y_{2 n}$.

Received by the editors May 26, 1994 and, in revised form, October 20, 1994.

1991 Mathematics Subject Classification. Primary 55P10.

This work was partially supported by the National Science Council of R.O.C.

(C)1996 American Mathematical Society 
Proof. Since the fibre of

$$
B g_{2 n}: \quad \mathrm{BO}(2 n) \rightarrow \mathrm{BSO}(2 n+1)
$$

is $\mathrm{SO}(2 n+1) / O(2 n) \cong R P^{2 n}$, the Becker-Gottlieb transfer [1] associated to this fibration is a stable map

$$
q_{2 n}: \quad \mathrm{BSO}(2 n+1) \rightarrow \mathrm{BO}(2 n)
$$

such that $\left(q_{2 n}\right)^{*} \circ\left(B g_{2 n}\right)^{*}$ is multiplication by the Euler charactertistic $\chi\left(R P^{2 n}\right)=$ $1(\bmod 2)$. Thus $q_{2 n}$ provides a stable splitting of $B g_{2 n}$, and

$$
\mathrm{BO}(2 n) \cong \mathrm{BSO}(2 n+1) \vee Y_{2 n} .
$$

This completes the proof.

Proof of Corollary B. This follows immediately from Theorem A and Lemma 1 by induction.

Lemma 2. There is a stable map $\lambda_{2 n}: \mathrm{BO}(2 n+2) \rightarrow \mathrm{BO}(2 n)$ such that the composite map

$$
\mathrm{BO}(2 n) \stackrel{B i_{2 n}}{\longrightarrow} \mathrm{BO}(2 n+2) \stackrel{\lambda_{2 n}}{\longrightarrow} \mathrm{BO}(2 n)
$$

is homotopic to the identity map, where the first map is the natural inclusion map.

Proof. This lemma follows immediately from [5] and [6].

Now we can prove Theorem A.

Proof of Theorem A. Let $g_{2 n}$ be the map $g_{2 n}(\alpha)=(\operatorname{det} \alpha) \oplus \alpha$. Then the diagram of groups

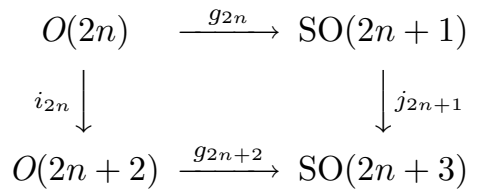

is strictly commutative, where $i_{2 n}$ and $j_{2 n+1}$ are the inclusions

$$
(\operatorname{det}(\alpha) \oplus \alpha) \oplus 1=\operatorname{det}(\alpha \oplus 1) \oplus(\alpha \oplus 1) .
$$

This yields a strictly commutative diagram of classifying spaces, and if $Y_{2 n}$ denotes the stable fibre of $B g_{2 n}$, then we have a commutative diagram of stable maps:

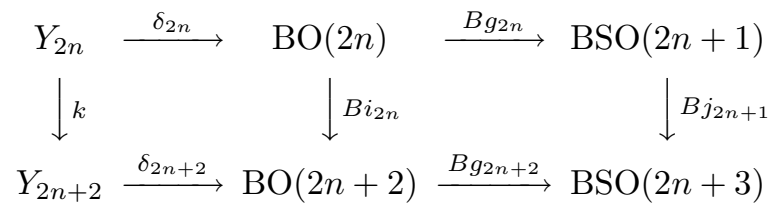

Now by Lemma $1, B g_{2 n}$ has a right inverse (in the stable category), that is, the fibration sequence splits, that is, $\delta_{2 n}$ has a left inverse, say $\eta_{2 n}$. By Lemma $2, B i_{2 n}$ has a left inverse, say $\lambda_{2 n}$. It follows that $k$ has a left inverse

$$
\left(\eta_{2 n} \lambda_{2 n} \delta_{2 n+2}\right) k=\eta_{2 n} \lambda_{2 n} B i_{2 n} \delta_{2 n}=\eta_{2 n} \delta_{2 n}=1 .
$$

This completes the proof of Theorem A. 


\section{REFERENCES}

1. J. Becker and D. Gottlieb, Transfer maps for fibrations and duality, Compositio Math. 33 (1976), 107-133. MR 55:9087

2. H. W. Henn and H. Mui, Stable splittings for classifying spaces of alternating, special orthogonal and special unitary groups, Contemp. Math., Amer. Math. Soc., Providence, RI, vol. 146, 1993, pp. 143-158. MR 94g:55021

3. S. Mitchell and S. Priddy, Symmetric product spectra and splittings of classifying spaces, Amer. J. Math. 106(1984), 219-232. MR 86g:55009

4. S. Mitchell and S. Priddy, Stable splittings derived from the Steinberg module, Topology 22 (1983), 285-298. MR 85f:55005

5. S. Mitchell and S. Priddy, A double coset formula for Levi subgroups and splitting $B G L_{n}$, Lecture Notes in Math., vol. 1730, Springer-Verlag, Berlin and New York, 1989, pp. 325-334. MR 90f: 55015

6. V. Snaith, Algebraic cobordism and K-theory, Mem. Amer. Math. Soc. 21 (1979), no. 221. MR 80k: 57060

Department of Mathematics, National Tsing Hua University, Hsinchu, Taiwan 30043 